\title{
Toward Pedagogy Driven Virtual Reality Learning Space Design
}

\author{
Erkki Rötkönen, A.K.M. Najmul Islam and Erkki Sutinen \\ University of Turku, Finland
}

\begin{abstract}
This paper argues for a need to develop Virtual Reality (VR) learning spaces based on pedagogy rather than implementing pedagogy in a general purpose VR application. In doing so, the paper summarizes the challenges in using an immersive virtual reality application to unite together primary school children across national, regional, and political boundaries in the same learning space in order to promote natural learning. Data was collected through observations during the activity sessions among children from a Finnish and a Bangladeshi primary school. Furthermore, we collected data using semistructured interviews and focus groups with teachers, heads of schools, and children from these two schools. The data analysis revealed three types of challenges: design, technological, and pedagogical. These findings guide us in designing and developing pedagogy centered learning spaces using virtual reality.
\end{abstract}

Keywords: Virtual Reality, Learning Environment, and Pedagogy.

\section{Introduction}

A new curriculum for compulsory basic education in Finland was implemented in 2016 in all schools. The goals for this development of skills are (1) to secure the necessary knowledge and skills as well as to encourage learning, (2) learning outside the classroom and by using technology, (3) transversal competences developed in all subjects, (4) familiarization with the fundamentals of programming, and (5) to have at least one interdisciplinary learning module a year. The curriculum also aims to promote 21st Century skills. For instance, skills for collaborative problem-solving and the use of information and communication technology (ICT) are also included in the new National Core Curriculum for primary and secondary education from 2016 onwards. In order to promote 21st Century skills in schools, new learning approaches are needed7. Even if there are technical or other challenges to the adoption of virtual reality (VR) within educational settings, VR applications can enhance learning and advance social and creative skills. The use of VR in learning situations seems to improve digital-age literacy, creative thinking, communication, collaboration and problem solving ability, which constitute the twenty-first century skills [10]. Instead

7 https://www.oph.fi/english/curricula_and_qualifications 
of the traditional way of learning where the teacher has the knowledge which is then distributed to the learner, a complementary approach to learning is the natural learning process where the learners can be actively engaged in the process and knowledge is constructed and distributed in a collaborative way [11].

The children from different countries are inspired to engage in activities within the shared space to experience the close-to-real-life perspectives that foster natural learning. By the term natural learning, we emphasize the learning opportunities through everyday real-life community activities [1]. However, currently available educational technologies, such as e-learning $[12,13]$ tools, or even traditional virtual reality platforms such as Second Life and Active Worlds, do not offer this kind of natural learning environment as these technologies lack immersion, the feeling of being there with another person, which is vital for providing close-to-real-life experience. In contrast, a natural learning space allows children to experience a fully immersive, multi-user 3D environment anywhere in the world using newly developed immersive technologies. The authentic feeling of being there together and the closeto-real-life interactions enabled by this technology could potentially revolutionize education and training.

From the research perspective, prior studies on the use of immersive learning spaces in primary education have been rare. Only a few studies used immersive virtual reality applications with children [5]. And to the best of our knowledge, no prior study united together children in an inclusive way across national, regional, and political boundaries in an immersive learning space. Most prior research focused on the use of immersive technologies in medical and surgical training [3] and professional training [2] among adults. The findings from these studies cannot be generalized to primary school children who are united together across national, regional, and political boundaries in the same learning space.

In order to fill the above practical, methodological, and research void, the aim of this paper is to find out the challenges of uniting together, in a common immersive virtual space, children from Finland and Bangladesh to promote natural learning in children through collaboration. We designed the learning environment using a general purpose (although immersive) VR application (e.g., Second Life) and Skype and applied pedagogy as most research tried to achieve in the past [4]. The observed challenges point to the need to develop learning spaces based on pedagogy rather than implementing pedagogy in a general purpose virtual reality platform.

\section{Description of the Learning Environment}

In this paper, we target to develop a shared learning environment by connecting diverse physical locations, contexts, and cultures. The learning environment should encompass but maybe also reshape the culture of the participating schools-their presiding ethos and characteristics, including how individuals interact with and treat one another-as well as the ways in which teachers may organize the educational setting to facilitate learning. 
The two schools participating in the virtual learning environment were both elementary schools (one from Finland and one from Bangladesh). Pupils from a $4^{\text {th }}$ grade class (aged between 9 and 11 years) and their classroom teachers from each school participated in this initiative. The two schools were about the same size. The Finnish school has 380 pupils on grades one to six, whereas, the Bangladeshi school had 420 pupils from grades one to eight. The Finnish school is governed by the municipality and the governance is based on the principle of decentralization, thus making the school responsible for practical teaching arrangements as well as the effectiveness and quality of the education provided. The Bangladeshi school is private and governed and funded by a Finnish NGO. Children from slums study in this school with free of cost.

The physical learning space in both schools consists of an ordinary classroom. The two classrooms are then connected through both a VR platform and Skype. The activities took place once a week and lasted 45 minutes at a time. The Skype connection was used to view live videos of the entire classrooms. Through VR, the children mainly tried two activities: 1) throwing a ball, 2) hide and seek. At a time, one child from each school could join the virtual space using the VR headset to perform the activities. The headset has a built-in headphone through which the pair can communicate with each other. In the "throwing a ball" activity, one child throws a ball and the other has to catch it. In the "hide and seek" activity, one child hides and the other child has to find him or her. The Skype connection was used for common classroom activities between the two participating classes. During the observed time period, those activities consisted mainly of learning simple words and phrases in both languages (Finnish and Bengali), e.g. greetings, introducing oneself, numbers and colors. Still when there was a technical problem that required the teachers' full attention and the pupils were without the teachers' active guidance, they sometimes started spontaneously interacting with each other by Skype, e.g. by dancing or playing some games with movements. Skype was also used by teachers to communicate before and after the classes as well as during the lessons whenever there was a problem with the VR or video/audio connection.

A snapshot of the learning situation is presented in Fig. 1 whereas, in Fig. 2 we present a snapshot of the VR application. 


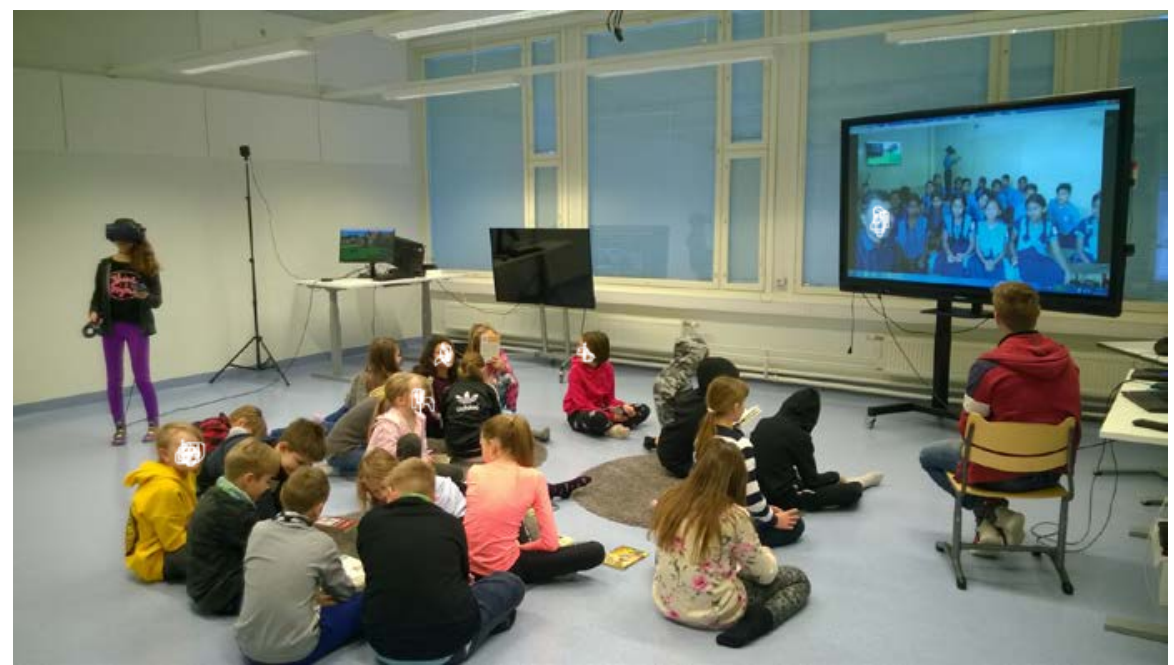

Fig. 1. A snapshot of Finnish classroom (the big screen runs Skype, the computer and screen on the left run the VR application)

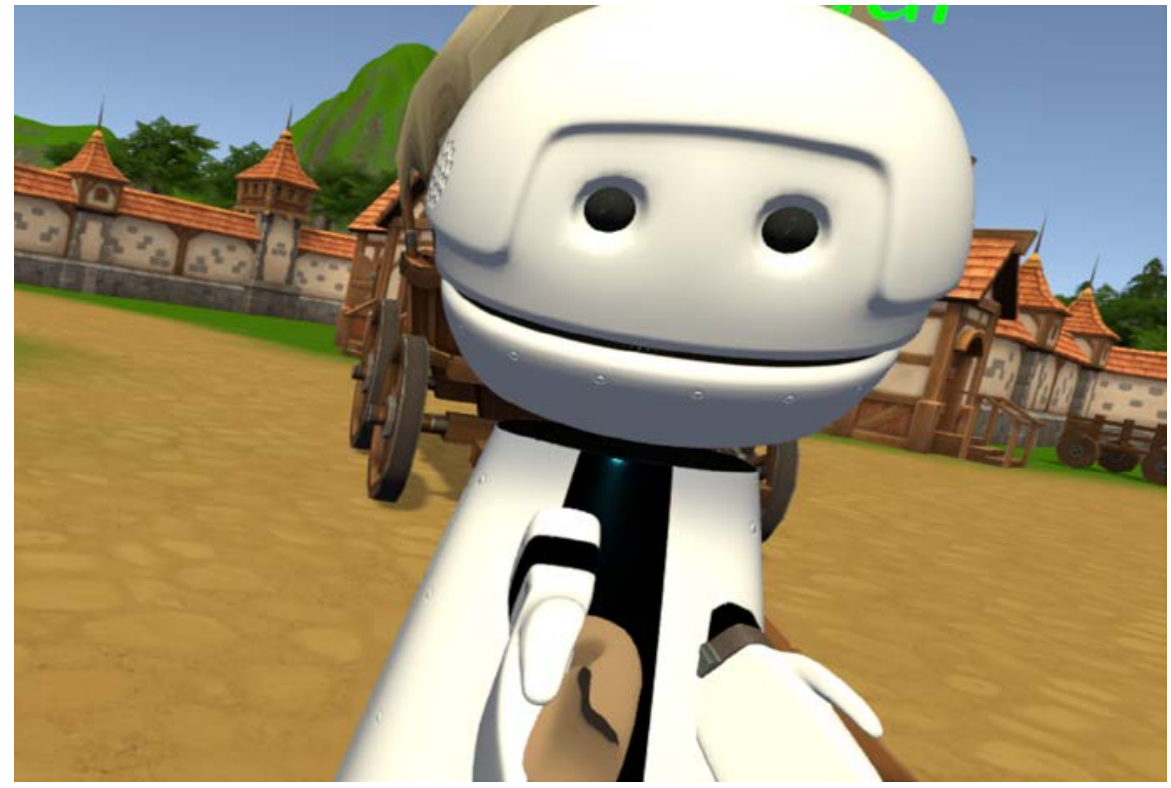

Fig. 2. A snapshot of the VR application (the pair of children is shaking hand) 


\section{$3 \quad$ Methodology}

\subsection{Data Collection}

In this study, we utilized a qualitative approach to examine the use of VR and Skype during the lessons. Before data collection, we obtained ethical approval from the city of Turku as well as in Bangladeshi school. Furthermore, we collected consent from the parents. We collected data using three approaches. First, one researcher was physically present at the Finnish school for doing observations during eight sessions from September 2018 to February 2018. The same researcher observed two sessions being physically present in the Bangladeshi school in February 2018. During the observations, the researcher took field notes as well as photos and videos of different activities. The observations helped the researchers observe, identify and understand different types of practical challenges that are faced during the sessions. Secondly, we collected data by interviewing two teachers from both schools as well as the ITperson in the Bangladeshi school using in-depth semi-structured interviews. The interviews lasted from 30 minutes to 60 minutes. Finally, two focus groups were arranged with the pupils. One focus group consisted of six Finnish children and the other focus group consisted of five Bangladeshi children. The purpose of the interviews and focus groups was to deepen our understanding on the issues that were found from the observations. The participants were interviewed in their own mother tongues. The interview protocol consisted of inquiring the following: participants' feelings and experiences, feedback and evaluation as well as suggested improvements. The teachers were also asked about their educational and professional background and their IT-skills. All the interviews were also recorded in the form of an audiotape and later transcribed. All the collected data has been summarized in Table 1.

Table 1. Collected data

\begin{tabular}{|c|c|c|}
\hline Method & Amount of data & Type of data \\
\hline Observations & $\begin{array}{l}\text { Observations in } 10 \text { sessions. } 8 \text { sessions } \\
\text { were observed being physically present in } \\
\text { the Finnish school and } 2 \text { sessions were } \\
\text { observed being in the Bangladeshi school. } \\
\text { Each session ran for } 45 \text { minutes. }\end{array}$ & Observation notes \\
\hline Interviews & $\begin{array}{l}4 \text { interviews among four teachers (two in } \\
\text { both countries) and one with the IT-person } \\
\text { in Bangladesh }\end{array}$ & $\begin{array}{l}\text { Audiotaped (later } \\
\text { transcribed), and } \\
\text { interview notes }\end{array}$ \\
\hline Focus groups & $\begin{array}{l}2 \text { focus groups among pupils, one in } \\
\text { Finland and the other in Bangladesh }\end{array}$ & $\begin{array}{l}\text { Audiotaped (later } \\
\text { transcribed and notes }\end{array}$ \\
\hline
\end{tabular}




\subsection{Data Analysis}

The data was content analyzed, which refers to a research technique for making replicable and valid inferences from texts (or other meaningful matter) to the contexts of their use [9]. First, the data was read several times to make sense of it as a whole and then broken into smaller units in order to assign codes. The codes were inductively developed. After that the codes are condensed to create categories.

\section{$4 \quad$ Results and Discussions}

The children had in general very positive attitudes toward the learning environment. Playing games, getting to know each other, communicating and creating together are exciting to them. Although some pupils were a bit shy when using English in Skype, it was easy to act in VR. The children found it fun that they do not only have to sit but they can do something active in VR. The pupils were excited about the feeling of being together in the same place even though they were on the different side of the world. Despite the positive attitudes, we observed several challenges from our data analysis. The challenges can be broadly classified into three categories: design challenges, technological challenges, and pedagogical challenges. These three types of challenges are very much interrelated with each other. In the following, we describe these challenges in detail and present evidence from the collected data.

\subsection{Design Challenges}

It is interesting to observe that the children felt that the design of the VR hardware and applications had been hindering their use. For example, when using VR, some of the children felt dizziness. They also found the cord in VR goggles was limiting their movements. The children came up with suggestions to have cordless goggles and smaller handles for children to make it easier to move and do different activities. A sample quote in the following highlights the issue:

"In my opinion some kind of children's controllers are needed so that it's easier for children to play in VR, so that those batons were smaller and that cord would be away so it would be some kind of Bluetooth"

The children also emphasized creativity, especially when building new environments and creating things together. However, at the same time the children wanted to have richer content with more real-life experiences. They wanted to see the other as she or he is, not just as an avatar, or at least have a profile picture of the other or a kind of a detector that could recognize the other. This finding is particularly interesting because most available virtual environments rely on avatars rather than real faces. Prior research suggested that avatars allow efficient interaction among learners in a common 3D virtual space [7]. At the same time, they can also use representational functions or artifacts with high fidelity [8]. In contrast, our findings clearly suggest that the children preferred real faces rather than avatars in the learning environment. Furthermore, they wish to meet, get to know each other, talk, shake hands, play and be together normally as in real life. In summary, they want to have 
more realistic interaction and activities. The following quotes indicate their expectations:

"So that one could see in that VR, when you put the goggles on, you could see that guy too not seeing him or her like avatar but a little like in that Skype thing so that there's we and them."

"There could be more people and so that it would feel like more real and as it is when you play in real life here. There could be like real house and day and night and it would feel more like real to do together."

\subsection{Technological Challenges}

Virtual reality technologies are new to the teachers involved in both schools. The Finnish teacher was more experienced in IT and could take part in building up and planning the used technology with the support of the VR solution provider but even to him the VR itself was new. Based on his IT skills, the Finnish teacher was in practice forced to take control of the technical aspects during the sessions and thus worked more with the IT person in Bangladesh than the classroom teacher. These technical issues also took the teacher's time from conducting the actual lesson. As the Bangladeshi teacher was afraid of the latest technologies like VR, the school decided to recruit an IT person to help with the technologies. However, it was interesting to observe that the IT person replaced the activities of teacher (e.g., communicating with the Finnish teacher, organizing sessions) and the original teacher became just an observer despite having basic computer skills. This issue was identified in the interview. A sample quote related to this is given below:

"I usually do lot of activities with computer such as word, excel, Internet searching. I also have computer at home. But VR is so unfamiliar to me that I did not feel very confident with it."

The VR solution provider organized short training for teachers and provided user guide. However, the training deemed insufficient. Thus, there have been several technological challenges in the project. Due to the technical challenges, many sessions were completely canceled. Among the technological problems, the sound issue of the VR headset was the most frequent one. In most sessions, the teachers failed to hear each other through the VR headsets. The VR solution provider changed the computer as well as the VR headset at the Finnish school. But still the situation did not improve. The next speculation was that the sound problem is because the Bangladeshi school has been using Skype and VR software in the same computer. Thus, there might have been issues in Windows sound settings. Taking this into account, the school decided to use separate computers for Skype and VR. However, the problem still continued. The teachers felt that sometimes resetting the VR software as well as VR headset solved the problem. But it was completely random. Taken together, the technology is just too complex at this moment. The IT person from Bangladesh explained the situation as follows:

"Resetting helps sometimes. But it's completely random if the system works or not. If you are lucky it works. Today just before the session, I tested and everything 
worked properly. But during the session, you saw headset microphone was not working”

\subsection{Pedagogical Challenges}

We observed several pedagogical challenges. First, the teachers felt that the VR technology is at a very primary stage and thus, it didn't bring much benefit for the learning of children at this stage. Only one child could try the VR headset at a time and others needed to observe what was happening on the screen, particularly because the teacher was involved in solving the technical problems and could not concentrate on the pupils, who were not involved in the VR. Thus, all other children remain mostly too passive during the sessions. A comment from a teacher reflects this:

"The pupils get bored if they only can watch the VR..."

"Technical problems need to be solved so that you don't have to fight with them most of the lesson."

However, the children did come up spontaneously with own ideas for activities like dancing together and doing some games with hands. The teacher still felt a significant school hour is lost for the children. Due to this reason, the teachers felt less excitement about the use of these technologies in the classroom at this moment. However, they thought that the current technologies might be still useful if the group is small or if it would enable more participants at a time to join the VR. The following sample quote illustrates this:

"I think half of the children are really bored as they don't have any other activity. These children are not very excited. To me these technologies should be tried at the school but they are at the very early stage of development and I somehow feel that these pupils are the guinea pigs and I feel bad for them, maybe a smaller number of students can participate”

In fact, the children suggested to have more activities where one can cooperate, e.g. role playing games, survival games, more realistic activities and games where all could participate at the same time. In summary, they pointed out the importance of having diverse and creative activities. The finding reflects the need for engaged realistic and situated instructional design methods [6].

Secondly, cooperation between the teachers is critical for successful pedagogical interventions during the sessions. However, the lack of collaboration between teachers from Bangladesh and Finland was observed. As mentioned earlier, the original grade- 4 teacher from the Bangladeshi school did not have an active role and there were practical difficulties in getting contact between her and the Finnish teacher. The Bangladeshi school designated the IT person for collaborating with the Finnish teacher. However, still there was hardly any collaboration between the IT person and the Finnish teacher for planning the sessions. Thus, the most sessions usually ran in quite an unplanned way. A proper planning of and defining the activities during a session are critical to gain maximum benefits from the sessions, which was largely missing. The Bangladeshi teacher commented the following regarding the lack of cooperation: 
"We were told the our children would learn Finnish language from them. They know better how to teach their language. So we had been always waiting for their instructions about what to do"

Finally, we observed a limited number of activities that can be conducted on the VR platform. The children mostly tried throwing a ball and hide \& seek activities. A carefully designed learning space with more engaging activities based on pedagogical consideration would be needed for maximizing the benefits. Several prior studies described the importance of learning situation that mimic real life interactive situation $[6,7]$. Several interviewees from both Finland and Bangladesh raised the issue regarding the lack of activities in the VR environment. Some of the sample quotes are as follows:

"The VR application has only 2-3 activities. It's easy to get bored, new interesting activities are needed, such activities that will be more interactive in nature "

"The activities may be ok at the initial stage, for just to know a bit more about the technology, but soon more activities and learning content is needed to keep the hype, otherwise it will quickly fade away"

It is a challenge to develop learning content that meets the 21st century skills through VR activities at this stage (of technologies). Thus, we plan to engage different stakeholders such as children, teachers, researchers, and designers in designing and developing the learning space as well as learning content through participatory approaches and by keeping pedagogy at the center. Furthermore, new approaches and further research are needed to carefully measure to what extent the learning outcome is or could be achieved.

Taken together, we observed that pedagogy needed to be the center of attention when developing a virtual learning space. This implies for a need to develop the VR learning space based on pedagogy rather than implementing pedagogy on a general purpose VR platform. We observed severe limitations of existing VR technologies supporting classroom education for children.

\section{Conclusion}

This paper reported the practical challenges of using an immersive virtual reality application to unite together children from two different countries in the shared learning space. We found the observed challenges are mainly design, technological and pedagogical in nature. Taken together, we argue for pedagogy-driven learning space development, rather than implementing pedagogy in a general purpose VR platform. We offer the following directions for future research and development based on our findings. First, the technical learning environment needs to be codesigned and developed by involving teachers, learners, pedagogical experts, designers, and researchers. The expectation for a natural rather than an avatar-based VR encounter calls for using remote presence technologies in learning space. Pedagogical considerations are needed to be at the center of attention. Secondly, future research should investigate what kinds of activities promote 21st century skills and how the learning outcome can be measured to evaluate the success of the 
activities as well as the learning environment. Thirdly, an experimental shared learning milieu between two different cultural settings might trigger novel learning affordances and skills that have not yet been identified in the well-known list of the 21st century skills: the researchers should be sensitive to recognize emerging opportunities that might revolutionize the learning process from how we conceptualize and comprehend the process. Last, teachers and children working in an experimental, research-focused learning milieu that makes use of and pilots novel technology need to be informed of their role as pioneers and co-designers; an apparently idle time in a classroom is a necessary component of any real-life, natural research environment.

\section{Acknowledgements}

We want to acknowledge our partners in this project: the city of Turku, Fida International, and Hesburger for their support in providing devices, classrooms and students as well as for funding and promoting this research. We would also like to thank all the children's parents for their support.

\section{References}

1. Dunst, C.J., Bruder, M.B., Trivette, C.M., Hamby, D., Raab, M., and McLean, M. (2001). Characteristics and Consequences of Everyday Natural Learning Opportunities. TECSE, 21 (2), 68-92.

2. Dávideková, M., Mjartan, M., and Gregus, M. (2017). Utilization of Virtual Reality in Education of Employees in Slovakia. Procedia Computer Science, 113, 253-260.

3. Yoganathan, S., Finch, D. A., Parkin, E., and Pollard, J. (2018). 360 Virtual Reality Video for the Acquisition of Knot Tying Skills: A Randomized Controlled Trial. International Journal of Surgery, 54, 24-27.

4. Mantziou, O., Papachristos, N. M., and Mikropoulos, T. A. (2018). Learning activities as enactments of learning affordance in MUVEs: A review-based classification. Educational Information Technologies, 23, 1737-1765.

5. Chiu, F. Y. (2017). Virtual reality for learning languages based on mobile devices. In IEEE 16th International Conference on Information Technology Based Higher Education and Training, 10-12 July, Ohrid, Macedonia.

6. Davis, T. (2012). Affordances of virtual worlds to support STEM project-based learning. In R., Capraro, M. Capraro \& Morgan, J. STEM Project-Based Learning: An Integrated Science, Technology, Engineering, and Mathematics (STEM) approach (pp. 77-84). USA: Sense publishers.

7. Pellas, N., Kazanidis, I., Konstantinou, N., and Georgio, G. (2017). Exploring the educational potential of three-dimensional multi-user virtual worlds for STEM education: A mixed-method systematic literature review. Educ Inf Technol, 22, 2235-2279.

8. Okutsu, M., DeLaurentis, D., Brophy, S., \& Lambert, J. (2013). Teaching an aerospace engineering design course via virtual worlds: A comparative assessment of learning outcomes. Computers \& Education, 60 (2), 288-298.

9. Krippendorff, K. (2004). Content analysis: An introduction to its methodology. Sage Publications: Thousand Oaks.

10. Papanastasiou, G., Drigas, A., Skianis, C., Lytras, M., \& Papanastasiou, E. (2018). Virtual and augmented reality effects on K-12, higher and tertiary education students' 
twenty-first century skills. Virtual Reality, 1-12. https://doi.org/10.1007/s10055-0180363-2.

11. McDonough, D. (2013). Similarities and Differences between Adult and Child Learners as Participants in the Natural Learning Process. Psychology, 4(3), 345-348. https://doi.org/10.4236/psych.2013.43A050.

12. Islam, A. N. (2015). The moderation effect of user-type (educators vs. students) in learning management system continuance. Behaviour \& Information Technology, 34(12), 1160-1170.

13 Islam, A. K. M. (2012). The role of perceived system quality as educators' motivation to continue e-learning system use. AIS Transactions on Human-Computer Interaction, 4(1), 25-43. 